\title{
El control de la vida personal, social y económica: desarrollo y estudio exploratorio de una escala de medida*
}

\author{
The Control of Personal, Social and Economic Life: \\ Development and Exploratory Study of a Scale of Measure
}

Recibido: julio 20 de 2011 | Revisado: febrero 2 de 2012 | Aceptado: febrero 26 de 2012

\author{
Maria Eugenia Morante Benadero*** \\ Miguel Costa Cabanillas $* * *$ \\ María Mercedes Esteban y PeÑA ***** \\ Centro de Promoción de Hábitos Saludables Madrid Salud.
}

Ayuntamiento de Madrid, España

\section{RES UMEN}

En este trabajo se presenta un estudio exploratorio sobre las características psicométricas de un instrumento sobre el control de de la vida personal, analizando su estructura factorial mediante un análisis exploratorio y confirmatorio de sus dimensiones. El tamaño del estudio final fue de 8.504 entrevistas y el criterio básico de estratificación fue el distrito municipal en 21 estratos. Los resultados encontrados señalan que los ítems en cada una de las dimensiones y sus pesos factoriales son aceptables y se relacionan con enunciados genéricos sobre la atribución de control. A su vez, los índices de bondad de ajuste encontrados de cada uno de los modelos propuestos, arrojan información interesante sobre las posibilidades que este cuestionario ofrece.

Palabras clave autores

Control de vida personal, estudio exploratorio, estudio confirmatorio.

Palabras clave descriptores

Control de vida personal, psicometría, confiabilidad, validez.

\section{A B S T R A C T}

The purpose of this study was to examine the characteristics psychometrics of a scale on the control of the personal life, analyzing its factorial structure by means of an exploratory and confirmatory analysis of its dimensions. The size of the final study was 8.504 interviews and the basic criterion was of stratification was the municipal district in 21 strata. The results indicate that the items in each of the dimensions and his weight factorials are acceptable and are related to generic terms of reference on the attribution of control. Also, the opposing indexes of goodness of fit of each one of the proposed models, throw interesting information about the possibilities that this scale offers.

Key words authors

Control of personal life, exploratory analysis, confirmatory analysis.

Key words plus

Control of Personal Life, Psychometry, Reliability, Validity. 


\section{Introducción}

Resulta difícil encontrar un concepto tan fértil y sugerente en la literatura psicológica como el de control. Desde que en la década de los sesenta Julian B. Rotter acuñara este término para referirse a las expectativas generalizadas de reforzamiento (Rotter, 1966), han aparecido otros términos con significados similares.

Rotter (1975) propuso que la percepción de control está basada en la contingencia perfecta entre un comportamiento y su resultado. En este sentido, y teniendo en cuenta la diferencia de la percepción de la contingencia del reforzamiento, Rotter (1966) desarrolló el concepto de control interno y externo. La importancia de este constructo radica en que una persona se anticipa a una situación dada de acuerdo con las expectativas generales que se ha formado según sus experiencias pasadas de reforzamiento, lo cual puede afectar una gran variedad de sus conductas. Estas expectativas generalizadas provocarán diferencias en las características de su conducta, y pueden actuar produciendo diferencias individuales dentro de una condición específica (Eisenberg, 1994). Según este mecanismo, si una persona interpreta la aparición del reforzador como dependiente de su conducta, el control es interno; si se percibe como independiente de su acción, el control es externo.

La percepción del control personal parece no depender exclusivamente de la contingencia entre el comportamiento y el resultado; implica también un conjunto de otros factores de tipo cognitivo y motivacional y, tal vez, existencial (Tamayo, 1993). Así, Skinner (1996) ha llegado a elaborar una guía de conceptos de control y estableciendo un marco conceptual para analizar más de 100 términos, tales como sentido de control, control primario, control secundario, autoeficacia, dominio, percepción de control, control percibido y tantos otros, con implicaciones teóricas, metodológicas y para la investigación. La revisión de Bullers (2000) también refleja la pluralidad del empleo de términos haciendo referencia al constructo control, y las diferentes teorías subyacentes al concepto. De este modo, etiqueta el constructo como control percibido, y lo caracteriza como la creencia individual sobre la habilidad de influir sobre un determinado resultado o evitar un resultado no deseado.

El constructo locus de control aparece en los últimos años asociado significativamente al proceso salud/enfermedad (Krause \& Shaw, 2000). En este sentido, una amplia literatura indica que lo sentimientos de control personal están relacionados con la salud física y mental a través del curso de la vida (Bandura, 1995). Más específicamente, la investigación sugiere que la gente que siente que puede influir en el curso de los eventos que afectan a sus vidas disfrutan de una mejor salud y tienen más expectativa de vida que los individuos que creen que el mundo externo no es influenciable a sus esfuerzos (Krause \& Shaw, 2000).

En el presente trabajo se parte de las escalas y cuestionarios que se han diseñado para medir esta sensación de influencia y se basan en preguntas orientadas a que las personas respondan si perciben o no esta sensación. Así, el propósito de este estudio es, precisamente, examinar la relación entre los sentimientos de control personal y, en resumen, la capacidad de producir resultados en el entorno a través de las propias acciones. El fundamento teórico de esta escala se encuentra en el concepto de locus de control propuesto por Rotter (1966) y el desarrollado a través otras múltiples investigaciones -tanto teóricas como experimentales-que han ido apareciendo en la literatura científica (Carton \& Nowicki, 1994; Ferguson, 1993; Phares, 1976; Weiner, 1991).

Así, en este trabajo se exponen las características psicométricas básicas del instrumento de Control de Vida Personal, analizando su estructura factorial mediante un análisis exploratorio y confirmatorio de sus dimensiones, y presentando, además, los datos referidos a la consistencia interna de sus dimensiones.

\section{Método}

\section{Procedimiento}

El estudio comenzó con un trabajo piloto para evaluar el tiempo y la dificultad de administración de la Encuesta de Salud de la Ciudad de Madrid de 2005 (ESCM '05). Durante la fase piloto no se detectaron 
problemas importantes. La información obtenida permitió introducir algunas modificaciones y estrategias para facilitar la presentación del cuestionario y aumentar la tasa de respuesta. Se realizaron 40 entrevistas ( 6 en menores de 16 años, 27 en adultos de 16 a 64 años y 7 en mayores de 65 años, correspondiendo el $45 \%$ a hombres y el $55 \%$ a mujeres).

El tamaño del estudio final fue de 8.504 entrevistas (7.341 adultos y 1.163 menores de 16 años). El criterio básico de estratificación fue el distrito municipal en 21 estratos. La asignación muestral por distrito fue prácticamente uniforme con un mínimo de 381 entrevistas. En cada distrito, se realizó una subestratificación por grupo de edad y sexo (menores de 16 años, 16 a 64 años y mayores de 65 años).

\section{Muestra}

Se ha utilizado como muestra únicamente los 7.341 adultos que formaron parte del estudio final. El criterio básico de estratificación fueron los 21 distritos municipales. La población de referencia de la ESCM '05 fue la población no institucionalizada, residente en Madrid e inscrita en el padrón de habitantes a fecha de 1 de septiembre de 2004, mes previo al lanzamiento del trabajo de campo. Esta población suponía un total de 3.162.304 habitantes. La participación fue voluntaria y toda la información recibida fue totalmente confidencial.

La edad media de los sujetos fue de 35 años y una desviación típica de 10.15 . El $54.7 \%$ de los participantes fueron mujeres. El $51 \%$ de los ciudadanos entrevistados informaron tener personal laboral activo, teniendo un $30 \%$ de este un contrato indefinido. En cuanto al nivel de estudios, cerca de un $30 \%$ tenía estudios secundarios y un $28 \%$, superiores. En cuando a la población inmigrante, el porcentaje se situó en torno al $13 \%$ del total de la muestra.

\section{Medidas}

\section{Control de Vida Personal}

El cuestionario contiene dos secciones claramente diferenciadas; la primera estudia el grado de control que tiene el individuo sobre diferentes aspectos de su vida personal y, la segunda, el grado de importancia que tiene cada uno de estos aspectos. Los ámbitos básicos estudiados fueron: relaciones con familiares, pareja, amigos, trabajo, economía, salud, ocio y tiempo libre, asuntos políticos y sociales.

El formato de respuesta de la primera parte del cuestionario es un continuo teórico con cinco categorías que van de 1 (No puedo influir en lo que ocurre) a 5 (Puedo influir en lo que ocurre).

\section{Resultados}

\section{Análisis factorial exploratorio}

La validez factorial del cuestionario se evaluó, en primer lugar, mediante un análisis factorial exploratorio. Previo al análisis, se utilizó el índice de adecuación de la muestra de Kaiser-MeyerOlkin (0.894) y la prueba de esfericidad de Bartlett $(p<0.0001)$, que mostraron una buena adecuación muestral y una apropiada correlación entre los ítems, indicando que los datos eran apropiados para la aplicación del análisis factorial.

Se utilizó el método de ejes principales y, dada la naturaleza relacionada de los factores, se aplicó una rotación oblimin directa. En el análisis se incluyeron los ítems de la primera sección, debido a que los de la segunda sección tienen exclusivamente la función de constatar el orden de importancia. El criterio de exclusión fue de 0.4 , es decir, que solo se consideraron aquellos ítems cuyos valores de saturación eran mayores al valor especificado. En la Tabla 1 se presenta la matriz de estructura obtenida en dicho análisis.

Tras el análisis factorial exploratorio, las estructuras factoriales obtenidas sugerían la posibilidad de considerar una estructura de tres factores como provisionalmente viable. El gráfico del Screen Test de Cattell (Cattell, 1966) o gráfico de sedimentación, mostró claramente una estructura tridimensional del test, presentando todos los ítems cargas factoriales superiores a 0.55 , criterio a partir del cual son consideradas como buenas (Comrey, 1973). El factor que más varianza explica es el Factor 1 (51.86 \%), mientras que el Factor 2 explica el $12.8 \%$ de la varianza y el Factor 3, el $5.6 \%$. 
TABLA 1

Matriz de cargas factoriales y varianza explicada

\begin{tabular}{cccc}
\hline \multirow{2}{*}{ Ítems } & \multicolumn{3}{c}{ Factor } \\
\cline { 2 - 4 } & $\mathbf{1}$ & $\mathbf{2}$ & $\mathbf{3}$ \\
\hline 1 & 0.59 & & \\
2 & 0.7 & & \\
3 & 0.73 & & \\
4 & 0.6 & & 0.81 \\
5 & & & \\
6 & 0.61 & & \\
7 & & 0.58 & \\
8 & & 0.71 & \\
\hline varianza explicada \% & 51.86 & 12.8 & 5.6 \\
\hline
\end{tabular}

Fuente: elaboración propia.

\section{Análisis factorial confirmatorio}

Complementariamente, se llevó a cabo un análisis factorial confirmatorio de los ítems para ratificar el modelo obtenido en el exploratorio. Se empleó el método de estimación de máxima verosimilitud (maximum likelihood) para analizar la matriz de correlaciones. Los análisis se llevaron a cabo mediante el programa de ecuaciones estructurales AMOS 5.0 (Arbuckle, 2003). Las varianzas de las variables latentes se fijaron en 1.0. Las varianzas de los términos de error fueron especificadas como parámetros libres.

Se contrastaron tres modelos diferentes. El modelo 1 se propone como hipótesis nula, y postula que existe un único factor en el que saturarían todos los ítems. El modelo 2 postula una estructura trifactorial, aunque con independencia entre los factores. El modelo 3 propone una estructura de tres factores que correlacionen entre sí. Los factores posibles serían Control de Vida Personal (ítems 1, 2, 3, 4 y 6), Control de Vida Económica (ítem 5) y Control de Vida Social (ítems 7 y 8).

Entre las diferentes medidas de bondad de ajuste que ofrece el programa, la más utilizada para verificar el ajuste del modelo es $\chi 2$ (chi cuadrado) dividido por los grados de libertad, aunque como proponen diferentes autores, para evaluar el ajuste entre el modelo teórico y el observado, no parece correcto emplear tan solo la prueba de chi cuadrado, ya que se encuentra afectada por el tamaño muestral (Bollen, 1989; Hayduck, 1987). En cuanto a su interpretación, se considera que un cociente de 4 es un ajuste razonable, mientras que aquellos valores cercanos a 2 son considerados como muy buenos. Otros indicadores de ajuste relativo son el índice de bondad de ajuste (GFI) y el índice de bondad de ajuste corregido a los grados de libertad del modelo (AGFI). Estos índices se encuentran entre los más utilizados, ya que están menos afectados por el tamaño de la muestra (Bentler, 1990). También se utilizó el índice de ajuste comparativo de Bentler (Comparative Fit Index [CFI]). Para que exista un buen ajuste, los valores GFI, CFI y AGFI deberían ser cercanos al valor de 0.9 (cuanto mayor sea el valor, mejor ajuste), al igual que el índice TuckerLewis Index (NNFI o TLI en AMOS 5.0).

Los índices de bondad de ajuste relativos al modelo que fue contrastado empíricamente están indicados en la Tabla 2.

Los resultados de los diferentes índices de ajuste utilizados confirman que el modelo 3, tres factores correlacionados, es el que mejor se ajusta a los datos. Puede observarse que los valores de CFI y GFI se sitúan cerca del valor 1.00 , mientras que los valores GFI, CFI, TLI y AGFI se sitúan muy cerca del valor 0.9 recomendado (Byrne, 2001). En cuanto al cociente $\chi 2 / g l$, se encuentra por debajo valor 2 , indicador de un ajuste excelente (Brooke, Russell \& Price, 1988).

\section{TABLA 2}

Índices de bondad de ajuste de cada uno de los modelos propuestos

\begin{tabular}{lccccc}
\hline \multicolumn{1}{c}{ Modelo } & $\chi^{2} / g l$ & GFI & AGFI & CFI & TLI \\
\hline 1. Un factor & 4.00 & 0.66 & 0.50 & 0.68 & 0.45 \\
\hline $\begin{array}{l}\text { 2. Tres factores } \\
\text { independientes }\end{array}$ & 3.75 & 0.85 & 0.70 & 0.88 & 0.75 \\
\hline $\begin{array}{l}\text { 3. Tres factores } \\
\text { correlacionados }\end{array}$ & 1.91 & 0.82 & 0.79 & 0.90 & 0.87 \\
\hline
\end{tabular}

(a) $\chi 2 /$ d.f.: chi-cuadrado dividido por los grados de libertad; GFI: índice de ajuste; AGFI: índice de bondad de ajuste; CFI: índice de ajuste comparativo; TLI: índice TuckerLewis.

(b) todos los valores del modelo son estadísticamente significativos a nivel $p<0.001$.

Fuente: elaboración propia. 


\section{Análisis de fiabilidad}

Para evaluar la fiabilidad de las escalas del cuestionario se ha realizado un análisis de consistencia interna a través del cálculo del coeficiente alfa de Cronbach. Los valores alfa se sitúan en torno a 0.7 y 0.8 , encontrándose dentro de los valores aceptables por Nunnaly y Bernstein (1994). La dimensión de Control Económico presenta el coeficiente de mayor fiabilidad (0.8), seguido por el de Control de Vida Personal (0.78) y Control de Vida Social (0.7).

\section{Discusión y conclusiones}

El objetivo principal de este trabajo era construir un instrumento válido y fiable de evaluación del control de vida personal, social y económica y, como hemos podido ver, el cuestionario aquí desarrollado se perfila como un instrumento de características psicométricas aceptables, comparable a otros instrumentos similares (Bowling, 1994). Tal y como se mostró en los resultados, los ítems en cada una de las dimensiones y sus pesos factoriales son aceptables y se relacionan con enunciados genéricos sobre la atribución de control. A su vez, los índices de bondad de ajuste de cada uno de los modelos propuestos arrojan información interesante sobre las posibilidades que este cuestionario ofrece.

La consistencia interna de cada una de las subescalas y del instrumento en su conjunto son adecuadas, lo que muestra que la escala mide con cierta precisión, es decir, que es susceptible para detectar diferencias interindividuales entre los sujetos investigados. No obstante, es importante plantear ciertas apreciaciones críticas.

Se ha considerado la conveniencia de realizar un posterior estudio acerca de las posibilidades predictivas del instrumento, es decir, una comprobación con datos empíricos que confirme o no que una medición precisa del locus de control mediante esta escala es relevante para predecir diferencias respecto a indicadores de salud. Además, si bien es cierto que el planteamiento inicial en la construcción del cuestionario se ha centrado en el apoyo social percibido, el presente trabajo ha llevado a la reflexión sobre la necesidad de integrar indicadores más objetivos en su evaluación.

Por todo lo visto, y a pesar de una cierta bondad, en lo que a los datos de fiabilidad y validez se refiere, parece necesaria una revisión del mismo que implique el replanteamiento de alguno de los presupuestos previos.

\section{Referencias}

Arbuckle, J. L. (2003). AMOS 5.0 update to the AMOS User's Guide. Chicago, IL: Smallwaters.

Bandura, A. (1995). Self-efficacy in changing societies. Cambridge: Cambridge University Press.

Bartlett, M. S. (1950). Test of significance in factor analysis. British Journal of Statistical Psychology, 3(2), 77-85.

Bentler, P. M. (1990). Comparative fit indexes in structural models. Psychological Bulletin, 107(2), 238-246.

Bollen, K. A. (1989). Structural equations with latent variables. New York: John Wiley \& Sons.

Bowling, A. (1994). La medida de la salud. Revisión de las escalas de medida de la calidad de vida. Barcelona: Masson.

Brooke, P. P., Jr., Russell, D. W. \& Price, J. L. (1988). Discriminant validation of measures of job satisfaction, job involvement, and organizational commitment. Journal of Applied Psychology, 73(2), 139-145.

Bullers, S. (2000). The mediating role of perceived control in the relationship between social ties and depressive symptoms. Women Health, 31(2-3), 97-116.

Byrne, M. (2001). Disseminating and presenting qualitative research findings. AORN Journal, 74(5), 731-732.

Carton, J. S. \& Nowicki, S. (1994). Antecedents of individual differences in locus of control or reinforcement: A critical review. Genetic Social and General Psychology Monographs, 120(1), 31-81.

Cattell, R. B. (1966). The meaning and strategic use of factor analysis. En R. Cattell (Ed.), Handbook of multivariate experimental psychology (pp. 174-243). Chicago: Rand McNally.

Comrey, A. L. (1973). A first course in factor analysis. New York: Academic Press.

Eisenberg, A. (1994). Influencia de la familia de origen en la orientación al logro y el locus de control. Tesis 
inédita de Maestría en Psicología, Universidad Nacional Autónoma de México, México DF, México.

Ferguson, E. (1993). Rotters locus of control scale -a 10-ítem 2- factor model. Psychological Reports, 73(3), 1267-1278.

Hayduck, L. A. (1987). Structural equation modeling with LISREL: Essentials and advances. Baltimore: The Johns Hopkins University Press.

Kaiser, H. F. (1970). A second generation little jiffy. Psychometrika, 35(4), 401-415.

Krause, N. \& Shaw, B. A. (2000). Role-specific feelings of control and mortality. En J. C. Nunnaly \& I. H. Bernstein (1994). Psychometric theory. New York: McGraw-Hill.

Nunnally, J. C. \& Bernstein, I. H. (1994). Psychometric theory (3a ed.). New York: McGraw-Hill.

Phares, E. J. (1976). Locus of control in personality. Psychology \& Aging, 15(4), 617-626.
Rotter, J. B. (1966). Generalized expectancies for internal versus external control of reinforcements. Psychological Monographs: General and Applied, $80(1), 1-28$.

Rotter, J. B. (1975). Some problems and misconceptions related to the construct of internal versus external control of reinforcement. Journal of Consulting and Clinical Psychology, 43(1), 56-67.

Skinner, E. A. (1996). A guide to constructs of control. Journal of Personality and Social Psychology, 71(3), 549-570.

Tamayo, A. (1993). Locus de control: diferencias por sexo y edad. Acta Psiquiátrica y Psicológica de América Latina, 39(4), 301-308.

Weiner, B. (1991). Metaphors in motivation and attribution. American Psychologist, 46(9), 921-930. 